\title{
III-V-Based Optoelectronics with Low-Cost Dynamic Hydride Vapor Phase Epitaxy
}

\author{
John Simon *, Kevin L. Schulte, Kelsey A. W. Horowitz, Timothy Remo, David L. Young and \\ Aaron J. Ptak
}

National Renewable Energy Laboratory, Golden, CO 80401, USA; kevin.schulte@nrel.gov (K.L.S.); Kelsey.Horowitz@nrel.gov (K.A.W.H.); timothy.remo@nrel.gov (T.R.); david.young@nrel.gov (D.L.Y.); aaron.ptak@nrel.gov (A.J.P.)

* Correspondence: john.simon@nrel.gov

Received: 27 September 2018; Accepted: 14 December 2018; Published: 20 December 2018

\begin{abstract}
Silicon is the dominant semiconductor in many semiconductor device applications for a variety of reasons, including both performance and cost. III-V materials exhibit improved performance compared to silicon, but currently, they are relegated to applications in high-value or niche markets, due to the absence of a low-cost, high-quality production technique. Here we present an advance in III-V materials synthesis, using a hydride vapor phase epitaxy process that has the potential to lower III-V semiconductor deposition costs, while maintaining the requisite optoelectronic material quality that enables III-V-based technologies to outperform Si. We demonstrate the impacts of this advance by addressing the use of III-Vs in terrestrial photovoltaics, a highly cost-constrained market.
\end{abstract}

Keywords: HVPE; III-V semiconductors

\section{Introduction}

Silicon as a semiconductor technology is beginning to run into significant technical limits. The death of Moore's Law has been predicted for decades, but there is now a clear evidence that transistor size limits have been reached, and improvements are only being realized through increases in complexity and cost. Si photovoltaics are also rapidly approaching the practical limit for solar conversion efficiency, with current best performance sitting at $26.6 \%$ [1]. In contrast to $\mathrm{Si}$, III-V materials, such as GaAs and GaInP have some of the best electronic and optical properties of any semiconductor materials. III-Vs have higher electron mobilities than $\mathrm{Si}$, enabling the transistors operating at high frequencies for wireless communication applications, and direct bandgaps, which permit extremely efficient absorption and emission of light. These materials appear, among other places, in power amplifiers (which enable the transmitting and receiving capabilities of cell phones), in high-value space-based photovoltaic (PV) panels, and in light emitting diodes (LEDs) for general illumination applications, with nitride-based III-Vs. III-V PV devices hold record conversion efficiencies for both single [2] and multijunction [1] solar cell devices, as well as one-sun modules [1]. Unlike Si, they can be quite thin, light, and flexible while maintaining a high conversion efficiency; they can reject heat, permitting them to operate at lower temperatures, in real-world outdoor conditions [3]; and they have lower temperature coefficients [4], resulting in a minimal performance degradation when their temperature does rise, which can reduce the requirements of heat sinking [3], and allow solar cells to be in intimate contact with rooftops. III-V materials are readily integrated in multijunction solar cell structures that increase efficiency, far beyond single junction limits. These qualities allow III-V PV modules to produce more energy than a similar power-rated silicon PV module, over the course of their lifetime [3]. 
The development of III-V materials and devices historically focused on quality, efficiency, and performance, with less regard to the cost of the epitaxial growth, and III-Vs lacked a driving force, like Si CMOS, to methodically push manufacturing costs to be significantly lower. So, while the performance of III-V devices is undeniably excellent, their cost has limited their use to applications that demand their high performance, or where the high cost of the manufacturing is amortized over the many devices grown in a single-batch deposition run. For example, thousands of LEDs, laser diodes, monolithic microwave integrated circuits, and heterojunction bipolar transistors are produced during one growth-run, in a production III-V reactor. In large-area applications like PV, where costs cannot be spread over numerous devices per batch, III-Vs are currently only used in niche, high-value (and low volume) markets, such as space power, concentrating PV in areas with high direct normal irradiance, and more recently, in area- and weight-constrained applications, like unmanned aerial vehicles (UAVs). If III-V materials were produced more cheaply than is possible, using today's manufacturing techniques, more widespread adoption of III-V's in PV and other opto-electronic applications could be achieved, and this increased market presence can further reduce manufacturing costs, similar to what was seen in Si cost reductions, as it expanded into various applications.

Thus, an innovative III-V manufacturing process is required. Hydride vapor phase epitaxy (HVPE) is a semiconductor growth technique that combines high epitaxial growth quality, high throughput, and high precursor material utilization. Several key features make HVPE more cost-effective than the current III-V epitaxial growth processes, including deposition rates as high as $300 \mu \mathrm{m} / \mathrm{h}$ for GaAs [5], the use of low-cost, elemental metal sources in the reaction, and a high utilization of the source materials, particularly hydride gases. HVPE was developed in the 1960s and was used commercially for the production of GaAsP LEDs, as well as photo-emitters and photo-detectors, for the telecommunications industry. HVPE largely fell out of favor, however, due to technical challenges not experienced by today's incumbent technologies, such as metal organic vapor phase epitaxy (MOVPE) and molecular beam epitaxy (MBE). Despite the obvious potential cost benefits delivered by HVPE, the high speed of the growth process and the residence times of the process gases, made it difficult to achieve the low-defect and chemically-abrupt heterointerfaces that are critical in many device structures.

To enable abrupt heterointerfaces vital to high performance III-V devices, but still maintain a high throughput, we developed a dynamic hydride vapor phase epitaxy (D-HVPE), a new route to low-cost III-V growth. D-HVPE enables the creation of abrupt heterointerfaces, while maintaining fast deposition rates. This can only be achieved in traditional HVPE by introducing detrimental growth interrupts. We designed a new HVPE reactor with multiple growth chambers, separated by inert gas curtains, shown schematically in Figure 1. In this approach, abrupt heterointerfaces are formed through the translation of a substrate from one growth chamber to another, each of which has an independently established, steady-state deposition reaction for the III-V material to be grown. This technique is effective at generating atomically- and chemically-abrupt interfaces [6,7]. This is the first step toward effective device formation at these high growth rates, and below we will discuss the optical and electrical properties of these interfaces. This two-chamber design takes advantage of the low-cost source materials (elemental metals) and high growth rates inherent to HVPE, while enabling abrupt, high quality interfaces. This research scale reactor uses a single $2^{\prime \prime}$ diameter substrate for simplicity, but future production scale reactors, like the ones assumed in the next section, will implement multiple, large-area substrates. The current growth system design approximates an in-line production reactor, in that the different device layers are deposited, as the substrate is shuttled through the various regions of a linear reactor. This design allows for the evaluation of the effectiveness of in-line device manufacturing, without the need to develop a fully in-line reactor. Employment of an in-line deposition process provides a pathway towards significant throughput increases and associated cost reductions, similar to how in-line deposition techniques already provide low-cost fabrication of thin-film PV devices, e.g., CdTe. Figure 2 shows a schematic of what the growth process will look like 
in a multi-chamber HVPE reactor that is capable of growing a high-efficiency single-junction GaAs solar cell in minutes, instead of the hours that it takes with the current manufacturing technology.

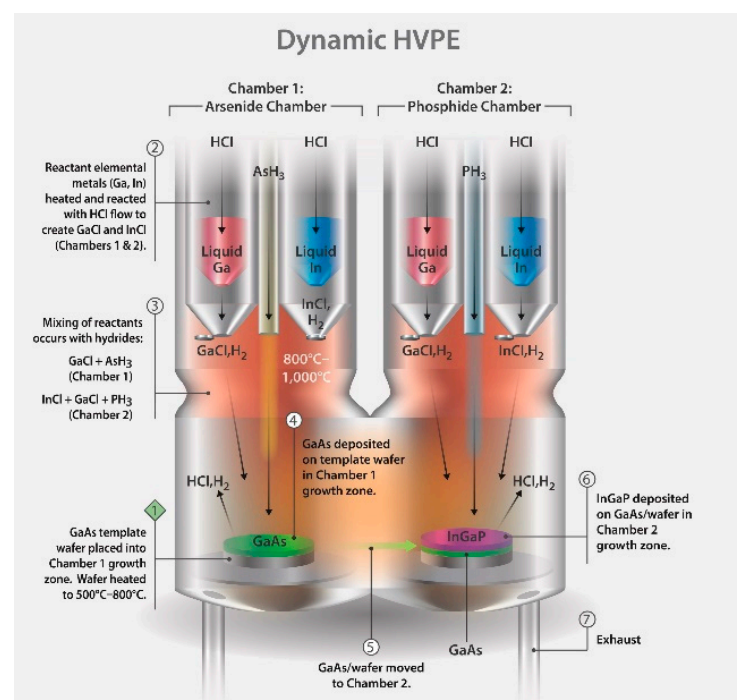

Figure 1. Schematic of the two-chamber dynamic hydride vapor phase epitaxy (D-HVPE) reactor at NREL, showing the side-by-side steady-state reactions for GaAs and GaInP. The substrate is rapidly shuttled between the growth chambers to create high-quality, chemically-abrupt interfaces.

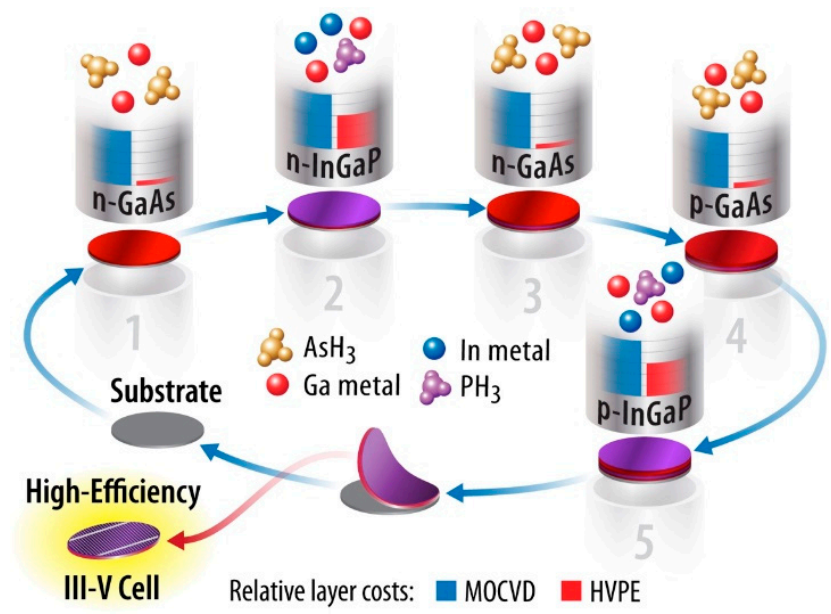

Figure 2. Schematic of the in-line HVPE reactor used to grow low-cost single junction GaAs solar cells, together with a substrate reuse technology.

In the next sections, we will examine the economic viability of D-HVPE and then demonstrated that this technique can be used to produce devices with a comparable performance to MOVPE grown devices. We developed a cost model for the HVPE growth of III-V devices, using a linear, in-line deposition system, conceptually similar to that shown in Figure 2. The cost analysis showed that it is possible for III-Vs to be dramatically less expensive than they are today. We focused on the specific case of using D-HVPE for the production of III-V-based solar cells, which could enable competitiveness in various cost-sensitive terrestrial PV applications, with market sizes that increase as substrate costs (which are concurrently the subject of intense research) are reduced. In the final section, we described the current efforts to produce defect-free interfaces and high-efficiency single-junction GaAs solar cells, using our custom D-HVPE growth system as an example of devices possible, using this technique. We demonstrated solar conversion efficiencies $>25.3 \%$ in the initial single-junction GaAs device grown at $\sim 60 \mu \mathrm{m} / \mathrm{h}$, similar to the efficiency of devices grown using the traditional growth technology. We also showed the initial results for the monolithic, HVPE-grown, two-junction GaInP/GaAs solar 
cell devices that incorporate three separate electrical junctions in a single device. The combination of low-cost growth, with the demonstration of high-efficiency devices, illustrates the promise of the D-HVPE technique, not only for solar cells, but for all III-V device applications.

\section{HVPE Deposition Costs}

We performed an analysis of the potential impact of the HVPE process on III-V deposition costs, using NREL's established bottom-up methodology [8-11]. For this analysis, we assumed that the NREL two-chamber HVPE reactor was scaled up to a high-volume, continuous reactor, with one zone for heating the substrate and one deposition zone per layer, as illustrated schematically in Methods Figure A1 in Appendix A. The deposition zones were isolated, using buffer sections incorporating inert gas curtains. As no such high volume HVPE reactor exists today, we created a basic model of the system to estimate throughput and cost per tool; this model was reviewed by members of the industry and their feedback was incorporated for accuracy. Details of the model appear in Appendix A and in Reference [12].

\section{Cost of the HVPE-Grown III-V Photovoltaic Devices}

We focused more specifically on the use of HVPE to produce low-cost, high-efficiency, III-V solar cells, and their potential competitiveness in different markets. If commercialized, D-HVPE could immediately provide value to PV markets that require high efficiency, high specific power, or flexible form factors (including consumer electronics, UAVs, the military, space markets, and automotive roofs [13]), by reducing the cost of epitaxy, while providing a performance similar to what is available today. The overall benefit depends on the cell type, production volume, and processes used for other aspects of the cell fabrication (e.g., metallization and choice of substrate), for a given manufacturer.

As discussed above, III-V solar cells at one-sun have not previously penetrated the mainstream PV power markets, due to their prohibitively high cost. In this section, we assess the potential for D-HVPE and substrate reuse to enable III-V technology, to compete in some of these markets, by modeling high volume costs for the III-V solar cells fabricated using these processes. We explore their potential balance-of-system (BOS) (e.g., racking materials and installation costs) and levelized the cost of energy (LCOE) (e.g., cost to produce a kWh of electricity) advantages over the incumbent $\mathrm{Si}$ flat-plate technology.

Our cell-cost models indicate that, at scale and with significant reductions in substrate cost, dual junction InGaP/GaAs cells deposited via HVPE could potentially reach costs below $\$ 0.50 / \mathrm{W}$, even with US manufacturing. This could allow these solar cells to be competitive in some broader PV markets that would benefit from the high-power density, low operating temperature and temperature coefficient, and the lightweight, flexible form factor, provided by III-V materials. Applications could include, for example, PV tile roofs, PV on electric vehicles (EVs), and certain residential and commercial rooftop installations that are weight- or area-constrained. In fact, at $<\$ 0.50 / \mathrm{W}$, III-V solar cells may even be competitive when dropped into the traditional PV module and system designs due to the ability of efficiency to reduce BOS costs in certain markets. In the remainder of this section, we explore the case of residential rooftop systems, which have higher BOS costs and areal constraints than the typical ground-mount, utility-scale installations, and thus, stand to benefit more significantly from the increased efficiency associated with III-V devices. We compared the total installed system cost and LCOE for the incumbent monocrystalline Si PERC [14] technology, to that of the HVPE-deposited III-V cells with a substrate reuse. PERC cells were chosen for comparison because they are rapidly gaining traction and are anticipated to become market-dominant over the next few years [15]. We used the modeled cell and module prices for both technologies that include the overhead costs and a sustainable product margin, rather than using current Si PERC cell prices, for the Si case, in order to obtain a technology-based comparison. The details and assumptions of this model appear in the methods section and Table A1 of Appendix A. The results are shown in Figure 3. While the III-V single junction cell costs are higher than that for the PERC cells, the increased efficiency of the III-V single junction 
cells, compared to Si results in the balance-of-module (BOM) (e.g., glass, encapsulant, busbars) and BOS cost savings, resulting in a comparable, total installed system costs. The savings was higher for the dual junction cells due to their higher efficiency, resulting in total installed system costs that could be comparable to those of the current PERC technology. At comparable installed system costs, III-V cells should provide a lower LCOE than $\mathrm{Si}$, due to the higher energy yield resulting from their lower operating temperature and temperature coefficient. In prior field measurements, single-junction GaAs cells exhibited 8\% higher energy yield than the Si cells in Phoenix, AZ in an open-rack configuration, though this varies with location [3]. Any increases in the energy production translate to decreases in LCOE; thus, III-Vs could have an 8\% lower LCOE, compared to the monocrystalline Si in Phoenix. The relative installed cost of the PERC cells compared to the HVPE-deposited III-Vs is similar for commercial rooftop systems, so similar LCOE reductions would also occur in these markets. Finally, the energy yield improvement and, thus, the LCOE benefit would be even greater in applications like solar shingles, where cells are direct-mounted onto the roof because of the lack of a suitable heat sink, for the cells.

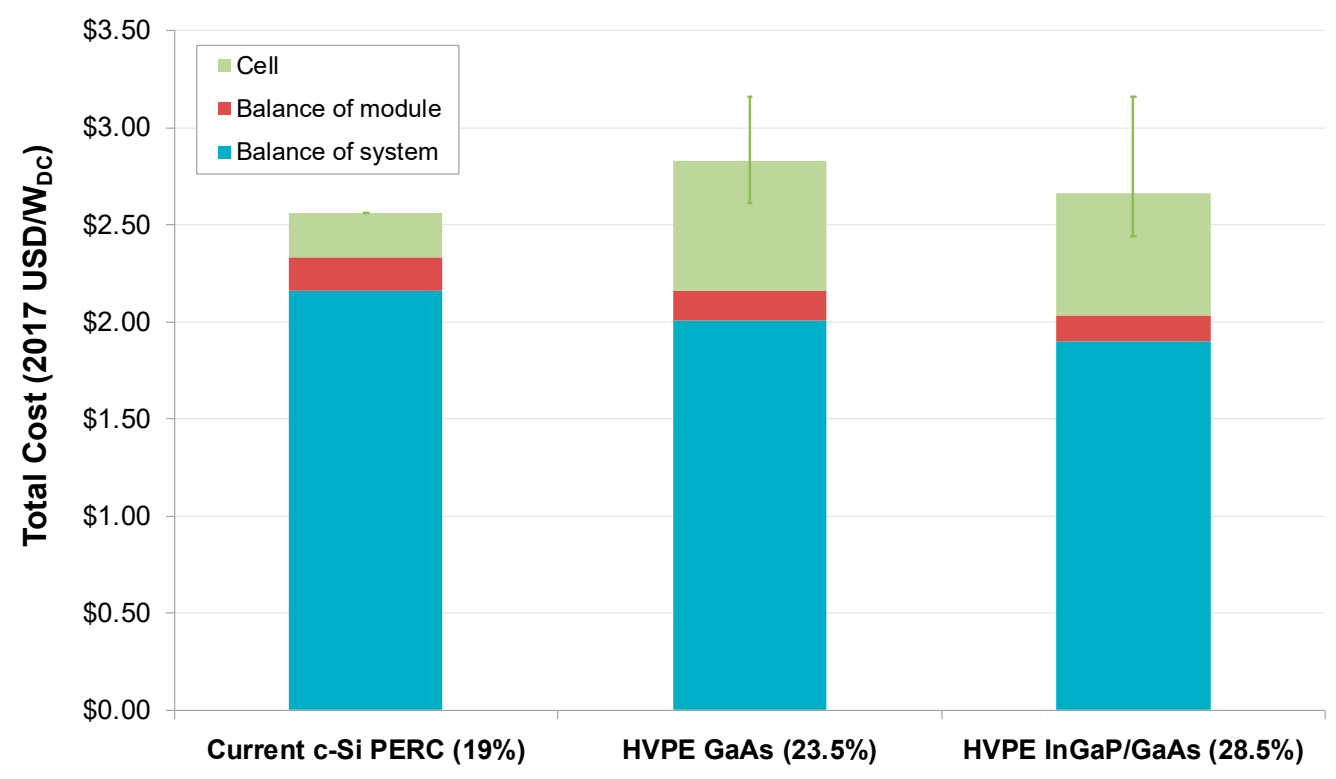

Figure 3. Comparison of total installed system costs for a $5.6 \mathrm{~kW}$ residential rooftop system for current monocrystalline Si PERC cells, compared to the estimates for D-HVPE-deposited GaAs and InGaP/GaAs cells, in the case where substrate costs are addressed. Assumes Chinese manufacturing and high-volume production (500 MW/year) for all cases. Cell, module, and system costs shown, include overhead costs and sustainable product margins.

The ability to reach costs on the order of $\$ 0.50 / \mathrm{W}$, depends critically on the cost of the substrate. These cost models assume that the cost of the substrate could be reduced, significantly, in the long-term to a value of $\sim \$ 1.00$ per $6^{\prime \prime}$ wafer, in this case, via a large number (approximately 100 , depending on the future GaAs substrate price) of substrate reuses and avoidance of a chemical-mechanical polishing (CMP) cost. The ability to reuse the substrate with limited or no CMP, through the use of a series of buffer layers has been demonstrated in the literature, but this has not yet been demonstrated at scale or with hundreds of wafer reuses [16,17], and this may be challenging to achieve with a high-yield. However, active research is taking place on substrate reuse, including chemical lift-off and mechanical fracture technologies, and lower cost substrates. To reach cell costs on the order of $\$ 1 / \mathrm{W}$ to $\$ 5 / \mathrm{W}$, which may be acceptable in some markets (e.g., PV on EVs, other portable power applications), less aggressive substrate cost targets would be sufficient. While there are III-V solar cell companies that currently implement the epitaxial lift-off [18] and reuse the substrate, it is unclear how many times the substrate is being reused. Additional research and development is required to demonstrate a 
hundred reuses, at scale with a high-yield and significantly reduced polishing and reclaim costs. The lift-off process itself must also be scaled-up; current production volumes for III-V cells are low. Alternatively, cost reductions could be achieved via the use of a low-cost substrate (e.g., a virtual substrate or template, direct-growth on a low-cost substrate), as long as similar efficiencies can be obtained, or a combination of the two (lower cost substrate that is reused a lower number of times).

The cost model presented here does assume the immediate implementation of the technology presented in this paper. In addition to the time to commercialize and scale-up the D-HVPE process, the substrate advances will require time and investments in research and development. However, because the results in Figure 3 are based on the 2017 module and system cost structures, and include modeled 2017 Si PERC cell costs, these comparisons essentially assume these III-V cell costs were achieved overnight. While this serves to illustrate the benefits of a higher III-V cell efficiency at the module and system level and provides a useful benchmark for understanding whether or not III-V cells might be applicable to general power markets, some additional discussion of the future is warranted. Further BOM and BOS cost reductions are anticipated in the future [19]. These reductions would benefit both $\mathrm{Si}$ and III-Vs, but the marginal value of higher efficiencies, and thus, the advantage of the III-V cells over $\mathrm{Si}$, would be somewhat reduced. Additionally, the efficiencies of $\mathrm{Si}$ systems are also anticipated to rise, although they are beginning to hit the practical limits [20], and Si module costs are expected to decrease, but will similarly asymptote, eventually, as the technology is already quite mature. We were not able to make quantitative comparisons of the installed system costs in these future scenarios due to the lack of PV cost-projections and general uncertainty around the future system cost structures. Finally, Figure 3 includes only the costs associated with single and dual junction III-V cells; HVPE could potentially allow for the addition of even more junctions at a low cost when scaled, increasing efficiencies further and enabling additional BOM and BOS cost savings that are out of reach for $\mathrm{Si}$.

\section{Development of D-HVPE for High Efficiency Devices}

HVPE was successfully employed in the past to produce commercial devices, such as LEDs and detectors, but the production of HVPE-grown III-V devices paled in comparison to the MOVPE grown devices [21,22], primarily due to the difficulty of making abrupt, highly-passivated heterointerfaces, as noted earlier. This is important because unpassivated interfaces have dangling atomic bonds or impurities that would otherwise act as non-radiative recombination sites and decrease the device performance. It is important that the potential low costs of the HVPE growth, detailed in the previous section, be viewed in the context of the achievable performance. The key test for D-HVPE is to effectively passivate III-V layers to decrease the carrier recombination at interface states, while still maintaining the high throughput that helps to make HVPE a low-cost technology.

A simple device structure that acts as a sensitive test of interface abruptness is the Esaki diode, or tunnel-junction [23]. Tunnel junctions are used in frequency converters, detectors, oscillators, amplifiers, and switching circuits. This diode uses a highly-doped n-type region, in intimate contact with a similar p-type region, in order to enable carrier tunneling from the valence band of one side of the junction to the conduction band of the other. The change from n-type to p-type must occur on sub-nanometer length scales to ensure significant wave-function overlap, making the observance of tunneling behavior a good measure of interface abruptness in these epitaxial layers. Our D-HVPE reactor allows us to form GaInP/GaAs heterointerfaces, both at a high growth-rate and without resorting to a detrimental pause in the growth process, for a change in material chemistry. Impurities can adsorb on the surface and native defects can form during growth interrupts, both of which lead to imperfect interface passivation, increased interface roughness, and detrimental device performance, such as, lack of tunneling, or a decreased solar conversion efficiency. In addition, interrupts reduce the throughput of the process, increasing device costs. Figure 4 shows the current-density vs. voltage characteristics of a tunnel junction grown via D-HVPE. This tunnel junction achieved a peak tunneling current of $11.2 \mathrm{~A} / \mathrm{cm}^{2}$, validating the ability of D-HVPE to form abrupt doping profiles. This device not only has an abrupt doping profile but also an abrupt material change, from GaInP to GaAs. 
The extremely low resistance across this tunnel diode (Figure 4 inset), allows us to use it in multijunction solar-cell structures to connect two subcells in series such that the voltages of the individual subcells add together to create a structure with a high solar conversion efficiency [24]. This indicates that D-HVPE is capable of creating heterojunctions that are thin, chemically abrupt, and free of detrimental defects that would lower the achievable performance necessary for a high-peak-current tunneling.

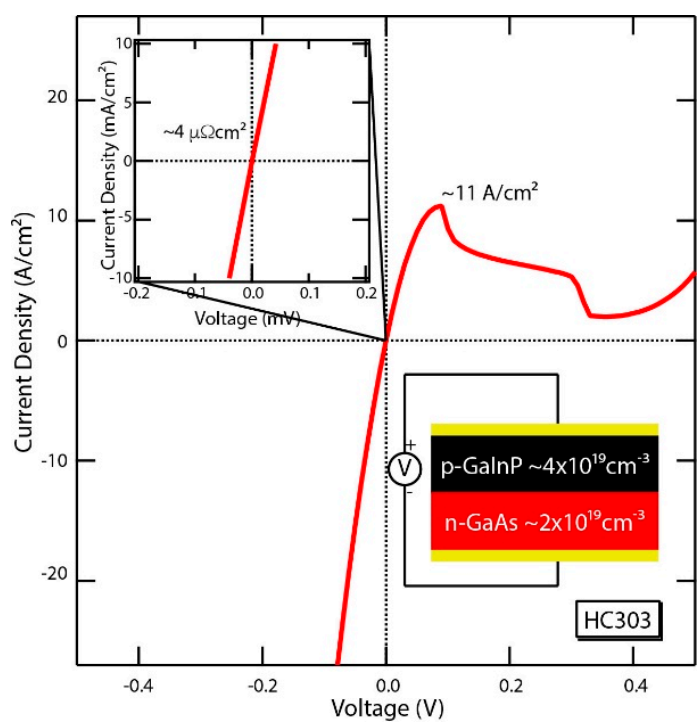

Figure 4. Current-density-voltage characteristics for a tunnel junction grown using D-HVPE. Inset shows an amplified look around $0 \mathrm{~V}$ showing the low resistance of the tunnel junction.

Solar cells also require passivated, low-defect interfaces to achieve a high performance, and cell performance provides an excellent evaluation of the material quality. For this reason, we grew a series of III-V solar cells to test the ability of D-HVPE to create interfaces that reduce minority carrier recombination. Figure 5 (left) shows a comparison of internal quantum efficiency (the ratio of incident, unreflected photons to those that are collected as a useful current) of single-junction GaAs solar cells with different levels of surface and interface passivation. The details of both the growth and the processing of these devices are available in Appendix A. The addition of front and back surface passivation (from solid red to black) directly improves the carrier collection at all wavelengths of light by minimizing the carrier recombination rate at these interfaces. Figure 5 also shows, for comparison, a MOVPE-grown GaAs solar cell with GaInP front and rear passivation that exhibits a nearly identical quantum efficiency to that of the D-HVPE-grown passivated device. Each of these passivated devices reach near-unity internal quantum efficiency, over a wide-range of wavelengths, from the GaAs band-edge at $\sim 890 \mathrm{~nm}$, until the GaInP passivating layers begin to absorb light below $\sim 670 \mathrm{~nm}$, meaning that every photon absorbed in this wavelength range is converted into a useful current. The nearly identical performance between the MOVPE and D-HVPE grown devices occurs despite the fact that the D-HVPE device growth-rate was an order of magnitude higher than that for the MOVPE control sample. This indicates that D-HVPE capably creates heterointerfaces, with an equal performance to that of the incumbent batch-growth technology, in a continuous process that can dramatically increase the III-V deposition throughput.

III-Vs also have the added benefit of easily incorporating additional alloy stacks to create multijunction devices that convert a larger portion of the solar spectrum even more efficiently. These devices are significantly more complicated, due to the larger number of layers and heterointerfaces needed in order for the device to operate properly. We previously showed GaInP top subcells with a good bulk material quality that can be coupled with the GaAs bottom subcell and the tunnel junction described here [24]. In order to demonstrate the viability of D-HVPE to manufacture more complex multijunction solar cell devices, with the potential for $>30 \%$ efficiency, we created a $\mathrm{GaInP} / \mathrm{GaAs}$ two-junction device with a GaInP/GaAs tunneling interconnect, to produce a monolithic 
two-terminal solar cell device. This device utilizes eight different layers (see Figure 6) that need to have the right composition, doping, and low-defect interfaces; a structure that would be nearly impossible to grow by traditional HVPE. Figure 6 shows the current density-voltage characteristics of a multijunction solar-cell device grown via D-HVPE, measured under one-sun illumination. The unpassivated top cell limits the current, however, the device still increases the $V_{O C}$ from the single-junction case from $1.08 \mathrm{~V}$ to $2.40 \mathrm{~V}$, highlighting the excellent bulk quality of both subcells. This device showcases the ability of D-HVPE to grow high quality devices that utilize multiple layers of different composition and doping, while maintaining high throughput. We expect that these devices, when properly optimized, can be grown in under $5 \mathrm{~min}$, as opposed to the multiple hours required by the MOVPE growth. We further expect that two-junction, D-HVPE-grown solar cells will yield conversion efficiencies close to 30\%, in the near future, with simple structural modifications [24], far in excess of the capabilities of the Si PV, with a growth technique that can approach the costs of the Si production.
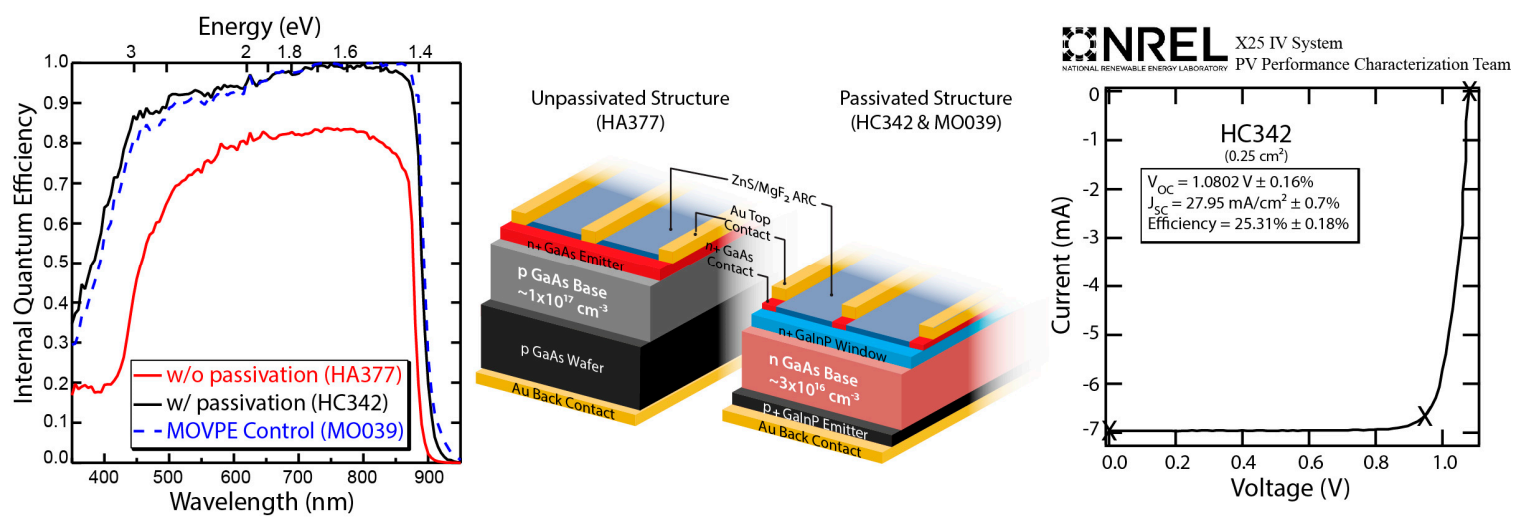

Figure 5. (Left) Internal quantum efficiency measurements of an unpassivated (red), and a fully passivated (black) single-junction GaAs solar cell, grown by D-HVPE at $\sim 60 \mu \mathrm{m} / \mathrm{h}$. The dashed blue curve represents data from an MOVPE-grown device with nominally the same structure (shown in the middle), but grown at $\sim 6 \mu \mathrm{m} / \mathrm{h}$. (Right) NREL-certified current-voltage data for the D-HVPE-grown GaAs solar cell with $25.3 \%$ conversion efficiency. It shows the current-voltage characteristics of a D-HVPE-grown single-junction GaAs solar cell, with front and rear passivation under simulated one-sun AM1.5G illumination. The device achieved a current density of $\sim 28 \mathrm{~mA} / \mathrm{cm}^{2}$, which matches the current density of the control MOVPE device grown with the same structure, and is only $6 \%$ lower than the record MOVPE device which utilized a more transparent AlInP window layer [2]. The open-circuit voltage $\left(V_{O C}\right)$, which can be used as a sensitive indicator of the crystal perfection in a solar cell, is $1.08 \mathrm{~V}$, only $0.04 \mathrm{~V}$ below the world-record MOVPE-grown devices [1].

All the device results presented here showed that D-HVPE enables III-V device growth, using this low-cost technique. We also expect the efficiency of the preliminary D-HVPE-grown solar cells, shown above, to increase with further optimization. For example, little work has been done to optimize the bulk material quality, through the use of optimized substrate temperature or reactant flow ratios, in order to decrease native or extrinsic defect concentrations. Additionally, the use of a more transparent AlInP window would create a significant positive increase on the current density, roughly $\sim 1.2 \%$ absolute, in a GaAs single junction solar cell, and $>30 \%$ in a multijunction device. The addition of an AlInP window would also result in a small increase in the $V_{O C}$. However, significant fundamental research is required to determine whether a high quality AlInP can be grown by HVPE. These improvements would lead to equal, or at least nearly-equal, device performance for a growth technique that projects to be much cheaper than the existing III-V technologies. 

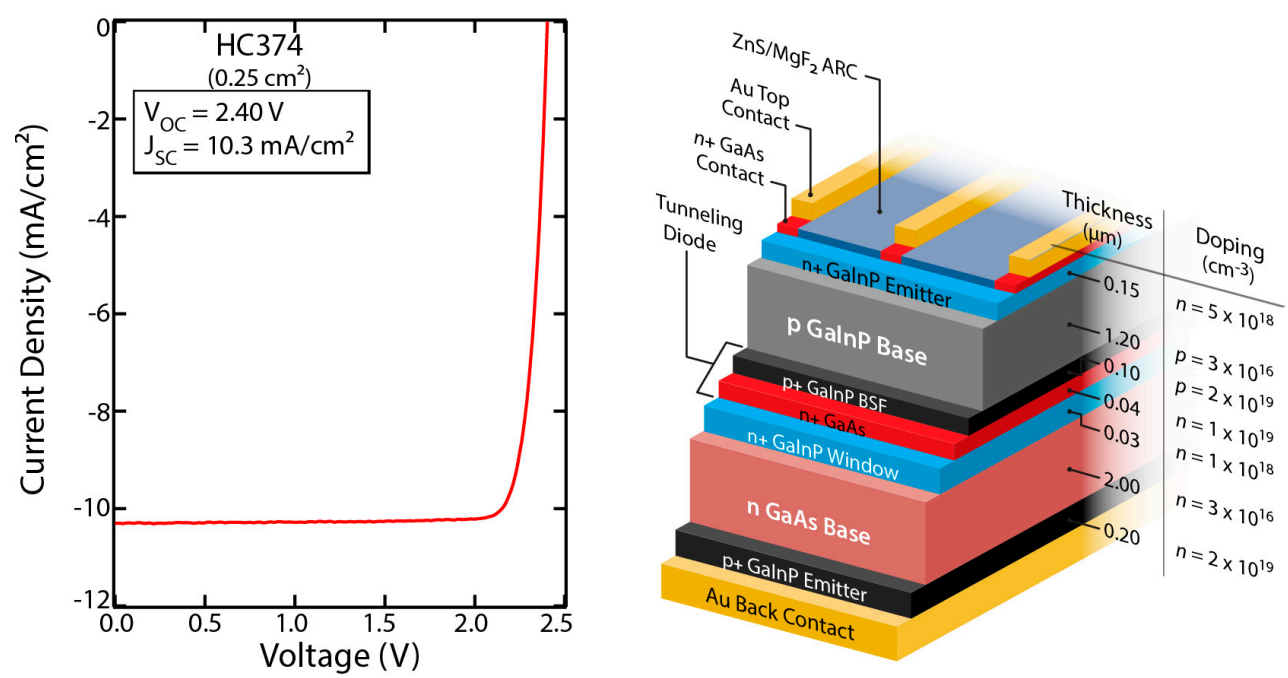

Figure 6. Current density-voltage characteristics for an HVPE-grown multijunction solar cell device under the AM1.5G solar spectrum. The structure of device is shown on the right.

While this section focused on the recent efforts to produce III-V solar cells, the D-HVPE technique is clearly applicable to many kinds of devices. HVPE is already used to produce high-quality nitride-based template layers, as well as $\mathrm{Ga}_{2} \mathrm{O}_{3}$, for power electronics applications. The use of the D-HVPE process for the formation of a more complicated device structures that rely on abrupt and electrically ideal interfaces, would allow for the production of low-cost, high-quality transistors, light emitters and detectors, and power conditioning devices, in addition to solar cells. Indeed, the development of low-cost III-V growth may enable technologies yet to be created.

\section{Conclusions}

We demonstrated the ability for D-HVPE to grow high-efficiency devices with a performance equivalent to that of conventional MOVPE, validating this new technology for the manufacture of high-performance III-V opto-electronic devices. We also developed a cost model for D-HVPE, in a high-volume production context. Our cost model further indicates that the use of D-HVPE, together with a low-cost substrate approach, could at last enable the use of III-V's in terrestrial PV markets, including certain residential and commercial rooftop installations. This technology could also be applied to manufacture non-PV devices, such as high-efficiency LEDs and devices for wireless communications applications.

Author Contributions: J.S. helped in designing and building the D-HVPE system, processed and helped characterize the devices presented here. K.L.S. helped in the design of the D-HVPE system and in the growth and characterization of the GaInP materials. K.A.W.H. helped develop the cost modelling. T.R. helped develop the cost modelling. D.L.Y. assisted in the design and building of the D-HVPE system. A.J.P. is the principal investigator of this work, helped in the design and building of the D-HVPE system, and contributed to the design and characterization of the tunnel junction devices. All authors contributed to the writing in this manuscript.

Funding: This work was authored by the Alliance for Sustainable Energy, LLC, the manager and operator of the National Renewable Energy Laboratory for the U.S. Department of Energy (DOE) under Contract No. DE-AC36-08GO28308. Funding provided for the cost model, tunnel junction, and multijunction cell work was provided by the Advanced Research Projects Agency (ARPA-E), US Department of Energy, award \#15/CJ000/07/05 and U.S. DOE Office of Energy Efficiency. Work on the single junction GaAs was provided by the U.S. DOE office of Energy Efficiency and Renewable Energy, Solar Energy Technologies Office. The views expressed in the article do not necessarily represent the views of the DOE or the U.S. Government. The U.S. Government retains and the publisher, by accepting the article for publication, acknowledges that the U.S. Government retains a nonexclusive, paid-up, irrevocable, worldwide license to publish or reproduce the published form of this work, or allow others to do so, for U.S. Government purposes.

Acknowledgments: The authors would like to thank David Guiling for materials growth and Michelle Young for device processing. 
Conflicts of Interest: The authors declare no conflict of interest.

\section{Appendix A.}

Appendix A.1. Methods

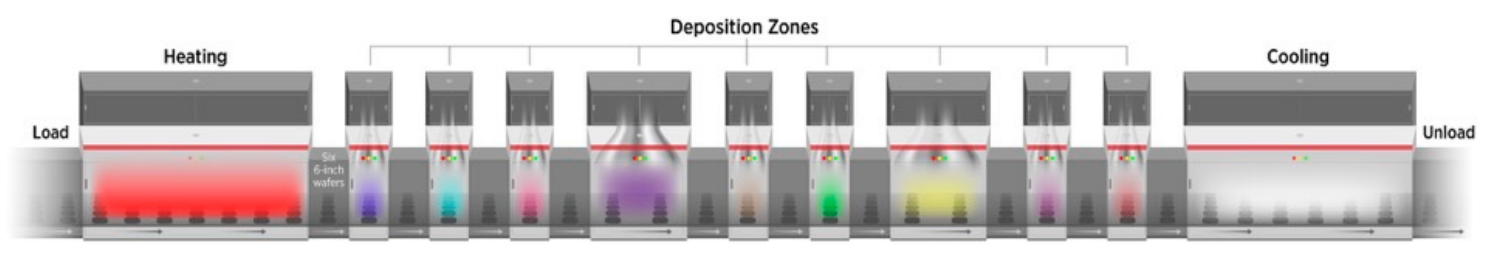

Figure A1. Diagram showing the concept of the high-volume, in-line HVPE tool used for cost analysis. In our model, there is one deposition zone, per layer, in the device.

\section{Appendix A.2. D-HVPE Reactor Cost Model}

In this model, the length of each deposition zone, $L$, is calculated as follows:

$$
L_{\text {zone } i}=\max \left\{\begin{array}{c}
D_{s} \\
\frac{t_{i}}{R_{i}} \cdot 60 \cdot S_{b}
\end{array}\right.
$$

where $t_{i}$ is the thickness of layer $i$ in $\mu \mathrm{m}, R_{i}$ is the deposition rate for layer $i$ in $\mu \mathrm{m} / \mathrm{h}, S_{b}$ is the reactor's belt speed in $\mathrm{mm} / \mathrm{min}$, and $D_{s}$ is the substrate diameter or width in $\mathrm{mm}$.

We calculated the length of the heating zone, similarly, except with $t$ equal to the required substrate temperature $\left(650^{\circ} \mathrm{C}\right.$ ) and $R$ equal to the heating rate (assumed to be $130{ }^{\circ} \mathrm{C} / \mathrm{min}$, based on heating rates currently observed in the laboratory reactor). The total length of the reactor is then equal to:

$$
L_{\text {total }}=L_{\text {heating }}+L_{\text {cooling }}+\sum_{i=1}^{N} L_{\text {zone } i}+N \cdot L_{\text {buffer }}+2 \cdot L_{\text {load } / \text { unload }}
$$

where $L_{\text {heating }}$ and $L_{\text {cooling }}$ are the lengths of the heating and cooling zones, respectively, $N$ is the number of deposition zones, $L_{\text {buffer }}$ is the length of the buffer zone between deposition zones, and $L_{\text {load/unload }}$ is the length required for loading or unloading. We assumed $L_{\text {buffer }}=0.3 \mathrm{~m}$ and $L_{\text {load/unload }}=1.5 \mathrm{~m}$. Here, we used a belt speed of $150 \mathrm{~mm} / \mathrm{min}$; this speed was within the range previously demonstrated for commercial vapor-phase deposition of the CdTe [25], and atmospheric pressure chemical vapor deposition (APCVD) of $\mathrm{Al}_{2} \mathrm{O}_{3}$ [26], of 12.7 to $381 \mathrm{~mm} / \mathrm{min}$. For the high-volume production, we assumed that there were six parallel 6" wafer tracks in the tool; similar numbers of wafer tracks have been used for commercial deposition of other thin film materials in the past. Belt speed, tool length, tool width, and deposition rate were free parameters that could be adjusted (within certain constraints) to optimize the design, when a commercial reactor is developed.

We estimated that the cost of each deposition chamber would be similar to that of a continuous APCVD system $[27,28]$, which sometimes also consist of gases flowing, vertically, through quartz reaction tubes onto a substrate conveyed along a belt. We collected data on single-chamber APCVD reactors via industry interview, and averaged these to obtain a base price, $P_{\text {base }}$, of $\$ 825,000$, excluding automation and auxiliary costs. For a scaled HVPE reactor with a similar width, we then approximated the total tool cost as:

$$
C_{\text {tool }}=P_{\text {base }} \cdot\left(\frac{\frac{\sum_{i=1}^{N} L_{\text {zone } i}}{N}}{L_{\text {base }}}\right)^{\alpha} \cdot(1+\beta \cdot(N-1))
$$

where $L_{\text {base }}$ is the length of the deposition area for a single chamber tool, used as a proxy for the HVPE costs, in this case a continuous APCVD reactor, $\alpha$ is a scaling factor for the tool price with length, and $\beta$ is a scaling factor representing the fractional price of each additional zone, compared to the base price. 
Both $\alpha$ and $\beta$ contain significant uncertainty at this point; however, based on interviews with several major suppliers of high-volume deposition equipment, we approximated the tool costs using $\alpha=0.75$ and $\beta=0.6$. Sensitivity analysis showed that the cost advantages of the HVPE deposition were robust to a range of $\alpha$ and $\beta$ values.

Key assumptions made in the deposition cost analysis are summarized in Table A1. Material price values were based on quotes received from material suppliers, interviews with members of industries that purchased the materials, and (where relevant) aggregation of data from online metal pricing sources and the US Geological Survey. All material pricing was based on the assumption of a high-volume production and large orders. For HVPE deposition, we assumed additional costs for automation and auxiliary equipment and installation equal to $22 \%$ and $20 \%$ of the total tool price, respectively. The material utilization rates for the MOVPE were based on prior work [10], and have been validated again by some industry members, as recently as 2018. Material utilization rates for the HVPE are currently uncertain. A Ga and In utilization of $70 \%$ was calculated from our research scale HVPE reactor. For all analysis, we assumed U.S. manufacturing and a 100\% plant capacity utilization.

\section{Appendix A.3. D-HVPE III-V Solar-Cell Cost Model}

We modeled the cell costs using the NREL cost model for single and dual junction III-V solar cells, under one sun illumination, first published in Reference [10]. The cost model was refined and updated in 2018, in order to reflect the current pricing for equipment and materials, as well as changes in process or capability. Figure 5 shows the device stacks modelled here. Low-cost metallization compatible with processing and epitaxial lift-off (ELO) of the III-Vs is still under development. Our cost model assumes the cell contacts are fabricated, using a low-cost plating, without the use of gold. We also assumed a high-volume ELO of the GaAs substrate and $\geq 100$ substrate reuses, without requiring chemical-mechanical polishing (CMP). The ability to reuse the substrate with limited or no CMP, through the use of a series of buffer layers has been demonstrated in the literature, but this has not yet been demonstrated at scale or with hundreds of wafer reuses [16,17], and this may be challenging to achieve with a high yield. However, active research is taking place on the substrate reuse, including chemical lift-off and mechanical fracture technologies, and a low-cost process with a high number of reuses may be feasible in the long-term.

Table A1. Key Input Assumptions for the HVPE Deposition Cost Model.

\begin{tabular}{cc}
\hline Input & Value \\
\hline Ga price $(6 \mathrm{~N})$ & $\$ 0.21 / \mathrm{g}$ \\
In price $(6 \mathrm{~N})$ & $\$ 0.82 / \mathrm{g}$ \\
High purity $\mathrm{AsH}_{3}$ price & $\$ 0.48 / \mathrm{g}$ \\
High purity $\mathrm{PH}_{3}$ price & $\$ 0.55 / \mathrm{g}$ \\
Ga material utilization & $70 \%$ \\
In material utilization & $70 \%$ \\
$\mathrm{AsH}_{3}$ material utilization & $30 \%$ \\
$\mathrm{PH}_{3}$ material utilization & $30 \%$ \\
$\mathrm{H}_{2}$ curtain flow rate & $10,000 \mathrm{sccm}$ \\
$\mathrm{HCl}_{\text {carrier gas flow rate }}$ Tool length & $14.5 \mathrm{sccm}$ \\
Tool price (including automation and auxiliary equipment) & $\$ 4.7 \mathrm{million}(1 \mathrm{~J}), \$ 11.6 \mathrm{million}(2 \mathrm{~J}$ cell) \\
Equipment maintenance cost & $4 \%$ of total equipment cost/year \\
\hline
\end{tabular}

We further assumed that III-V cells can be dropped into the standard modules employed for the c-Si cells. We used the NREL's module cost model to calculate the module cost and minimum sustainable price (MSP) (see References [8-11] for a description of MSP). The module MSP was input into the NREL's system cost models for residential, commercial, and utility scale systems [29] to evaluate the total system cost. 


\section{Appendix A.4. D-HVPE III-V Material Growth Methods}

All materials and devices shown in this work were grown in our dual chamber, Dynamic-HVPE (D-HVPE) reactor [30], using pure $\mathrm{Ga}$ and In metal, $\mathrm{HCl}, \mathrm{AsH}_{3}, \mathrm{PH}_{3}$, and $\mathrm{H}_{2}$ carrier gas. Dilute $\mathrm{H}_{2} \mathrm{Se}$ was the n-type dopant, while dimethylzinc was the p-type dopant. Heterointerfaces were formed by rapid mechanical transfer of the substrate between the two growth chambers, with each chamber stabilized at a new growth condition (either a change in material, doping, or both) for at least one minute before transfer. Substrates were (100) $n+$ GaAs doped with $\mathrm{Si}$ and offcut $4^{\circ}$ towards the (111)B plane. The growth rates of the GaAs absorber layers were $60 \mu \mathrm{m} / \mathrm{h}$, while the tunnel junction GaAs and GaInP layers were grown at $6 \mu \mathrm{m} / \mathrm{h}$. The GaInP absorber in the tandem device was grown at $54 \mu \mathrm{m} / \mathrm{h}$. An $\mathrm{n}+\mathrm{GaAs}$ buffer was grown to bury contamination at the initial growth interface, before device growth.

Solar cell devices were processed using the method detailed in Reference [31]. Unpassivated devices were processed on wafer, while passivated devices were grown inverted and removed from the wafer. First, a broad area Au contact was electroplated onto the back-contact layer. The Au surface was bonded to a Si handle, using an epoxy and the substrate was selectively etched away, using a $\mathrm{NH}_{4} \mathrm{OH} / \mathrm{H}_{2} \mathrm{O}_{2} / \mathrm{H}_{2} \mathrm{O}$ 1:2:2 solution, exposing a GaInP etch stop layer. This layer was removed, selectively, with hydrochloric acid. A grid pattern was defined by the standard lithography techniques, and $\mathrm{Ni} / \mathrm{Au}$ front contact grids were electroplated for the front grid contacts of all devices. Finally, $5 \mathrm{~mm}$ $\times 5 \mathrm{~mm}$ area devices were defined by lithography and isolated using selective wet chemical etching.

We measured solar cell external quantum efficiency (EQE) on a custom instrument, in which chopped, monochromatic light was split and then sent to the device of interest and a calibrated, broadband reference diode. We measured the output current of the device and reference on a lock-in amplifier, and used it to calculate the EQE, which is the ratio of electron current out to incident photons. Specular reflectance from the device surface was measured with a separate, calibrated reference diode.

We compared the measured EQEs to those of the calibrated GaInP and GaAs reference cells, to calculate the spectral correction factors for the AM1.5G spectrum. We set the height of a Xe-lamp solar simulator to obtain an illumination of $1000 \mathrm{~W} / \mathrm{cm}^{2}$, determined by measuring the current from the reference GaAs cell held under the lamp and adjusting by the spectral correction factor for the subcell of interest. We set dual junction top cell illumination by placing the GaInP reference cell, under the lamp at the GaAs one-sun height, and added current using a $470 \mathrm{~nm}$ LED to obtain one-sun equivalent illumination. The tandem cell one-sun current density-voltage curves were measured under the adjusted spectrum.

\section{References}

1. Green, M.A.; Hishikawa, Y.; Warta, W.; Dunlop, E.D.; Levi, D.H.; Hohl-Ebinger, J.; Ho-Baillie, A.W.H. Solar Cell Efficiency Tables (Version 50). Prog. Photovolt. Res. Appl. 2017, 25, 668-676. [CrossRef]

2. Kayes, B.M.; Nie, H.; Twist, R.; Spruytte, S.G.; Reinhardt, F.; Kizilyalli, I.C.; Higashi, G.S. 27.6\% Conversion efficiency, a new record for single-junction solar cells under 1 sun illumination. In Proceedings of the 37th IEEE Photovoltaic Specialists Conference, Seattle, WA, USA, 19-24 June 2011.

3. Silverman, T.J.; Deceglie, M.G.; Marion, B.; Cowley, S.; Kayes, B.; Kurtz, S. Outdoor Performance of a Thin-Film Gallium-Arsenide Photovoltaic Module. In Proceedings of the IEEE 39th Photovoltaic Specialists Conference (PVSC), Tampa, FL, USA, 16-21 June 2013.

4. Osterwald, C.R.; Glatfelter, T.; Burdick, J. Comparison of the Temperature Coefficients of the Basic I-V Parameters for Various Types of Solar Cells. In Proceedings of the Nineteenth IEEE Photovoltaic Specialists Conference, New Orleans, LA, USA, 4-8 May 1987.

5. Deschler, M.; Grüter, K.; Schlegel, A.; Beccard, R.; Jürgensen, H.; Balk, P. Very Rapid Growth of High Quality GaAs, InP and Related III-V Compounds. J. Phys. Colloq. 1988, 49, 689-692. [CrossRef]

6. Jain, N.; Simon, J.; Schulte, K.L.; Friedman, D.J.; Diercks, D.R.; Packard, C.E.; Young, D.L.; Ptak, A.J. Tunable Bandgap GaInAsP Solar Cells With 18.7\% Photoconversion Efficiency Synthesized by Low-Cost and High-Growth Rate Hydride Vapor Phase Epitaxy. IEEE J. Photovolt. 2018, 99, 1-7. [CrossRef] 
7. Schulte, K.L.; Simon, J.; Mangum, J.; Packard, C.E.; Gorman, B.P.; Jain, N.; Ptak, A.J. Development of GaInP Solar Cells Grown by Hydride Vapor Phase Epitaxy. IEEE J. Photovolt. 2017, 7, 1153-1158. [CrossRef]

8. Goodrich, A.; Hacke, P.; Wang, Q.; Sopori, B.; Margolis, R.; James, T.L.; Woodhouse, M. A wafer-based monocrystalline silicon photovoltaics road map: Utilizing k nown technology improvement opportunities for further reductions in manufacturing costs. Sol. Energy Mater. Sol. Cells 2013, 114, 110-135. [CrossRef]

9. Horowitz, K.A.; Woodhouse, M.; Lee, H.; Smestad, G.P. A Bottom-Up Cost Analysis of a High Concentration PV Module. In AIP Conference Proceedings; AIP Publishing: College Park, MD, USA, 2016.

10. Woodhouse, M.; Goodrich, A. A Manufacturing Cost Analysis Relevant to Single- and Dual-Junction Photovoltaic Cells Fabricated with III-Vs and III-Vs Grown on Czochralski Silicon. Available online: http: / /www.nrel.gov/docs / fy14osti/60126.pdf (accessed on 14 December 2018).

11. Woodhouse, M.; Jones-Albertus, R.; Feldman, D.; Fu, R.; Horowitz, K.; Chung, D.; Jordan, D.; Kurtz, S. On the Path to SunShot: The Role of Advancements in Solar Photovoltaic Efficiency, Reliability, and Costs. Available online: http:/ / www.nrel.gov/docs/fy16osti/65872.pdf (accessed on 14 December 2018).

12. Horowitz, K.A.W.; Remo, T.; Smith, B.; Ptak, A. Techno-Economic Analysis and Cost Reduction Roadmap for III-V Solar Cells; National Renewable Energy Lab. (NREL): Golden, CO, USA, 2018.

13. Audi Cooperates with Alta Devices on Automobiles with Solar Roofs. Available online: https://www. altadevices.com/audi-cooperates-alta-devices-automobiles-solar-roofs/ (accessed on 14 December 2018).

14. Green, M.A. The Passivated Emitter and Rear Cell (PERC): From conception to mass production. Sol. Energy Mater. Sol. Cells 2015, 143, 190-197. [CrossRef]

15. VDMA. International Technology Roadmap for Photovoltaic. 2017. Available online: http://www.etippv.eu/fileadmin/Documents/ETIP_PV_Conference_2017_Presentations/Session_II._1._Axel_Metz.pdf (accessed on 14 December 2018).

16. Ward, J.S.; Remo, T.; Horowitz, K.; Woodhouse, M.; Sopori, B.; VanSant, K.; Basore, P. Techno-economic analysis of three different substrate removal and reuse strategies for III-V solar cells. Prog. Photovolt. Res. Appl. 2016, 24, 1284-1292. [CrossRef]

17. Lee, K.; Zimmerman, J.D.; Xiao, X.; Sun, K.; Forrest, S.R. Forrest. Reuse of GaAs substrates for epitaxial lift-off by employing protection layers. J. Appl. Phys. 2012, 111, 033527. [CrossRef]

18. Yablonovitch, E.; Gmitter, T.; Harbison, J.P.; Bhat, R. Extreme selectivity in the lift-off of epitaxial GaAs films. Appl. Phys. Lett. 1987, 51, 2222. [CrossRef]

19. Cole, W.; Mai, T.; Richards, J.; Das, P. 2017 Standard Scenarios Report: A U.S. Electricity Sector Outlook. Available online: https:/ /www.nrel.gov/docs/fy18osti/68548.pdf (accessed on 14 December 2018).

20. Shockley, W.; Queisser, H.J. Detailed Balance Limit of Efficiency of p-n Junction Solar Cells. J. Appl. Phys. 1961, 32, 510-519. [CrossRef]

21. Gale, R.P.; McClelland, R.W.; King, B.D.; Fan, J.C.C. Thin-film solar cells with over $21 \%$ conversion efficiency. Sol. Cell 1989, 27, 99-106. [CrossRef]

22. Usui, A.; Matsumoto, T.; Inai, M.; Mito, I.; Kobayashi, K.; Watanabe, H. Room Temperature cw Operation of Visible InGaAsP Double Heterostructure Laser at $671 \mathrm{~nm}$ Grown by Hydride VPE. Jpn. J. Appl. Phys. 1985, 24, L163. [CrossRef]

23. Esaki, L. New Phenomenon in Narrow Germanium $p-n$ Junctions. Phys. Rev. 1958, 109, 603-604. [CrossRef]

24. Schulte, K.L.; Simon, J.; Ptak, A. Multijunction GaInP/GaAs Solar Cells Grown by Hydride Vapor Phase Epitaxy. Prog. Photovolt. 2018, 26, 887-893. [CrossRef]

25. Kaydanov, V.I.; Ohno, T.R. Process Development and Basic Studies of Electrochemically Deposited CdTe-Based Solar Cells. Available online: http:/ /www.nrel.gov/docs/fy01osti/29956.pdf (accessed on 14 December 2018).

26. Black, L.E. New Perspectives on Surface Passivation: Understanding the $\mathrm{Si}-\mathrm{Al}_{2} \mathrm{O}_{3}$ Interface; Springer: Basel, Switzerland, 2016.

27. Chemical Vapor Deposition. Available online: http://users.wfu.edu/ucerkb/Nan242/L10-CVD_b.pdf (accessed on 14 December 2018).

28. Williams, J.D. Microfabrication and Semiconductor Processes. Available online: http://www.slideshare.net/ maheeniqbal1420 / part-6-thin-film-depositoin (accessed on 14 December 2018).

29. Fu, R.; Feldman, D.; Margolis, R.; Woodhouse, M.; Ardani, K.U.S. Solar Photovoltaic System Cost Benchmark: Q1 2017. Available online: https://www.nrel.gov/docs/fy17osti/68925.pdf (accessed on 14 December 2018). 
30. Young, D.L.; Ptak, A.J.; Kuech, T.F.; Schulte, K.; Simon, J.D. High Throughput Semiconductor Deposition System (No. 9,824,890); National Renewable Energy Lab. (NREL): Golden, CO, USA, 2017.

31. Duda, A.; Ward, J.S.; Young, M. Inverted Metamorphic Multijunction (IMM) Cell Processing Instructions (No. NREL/TP-5200-54049); National Renewable Energy Lab. (NREL): Golden, CO, USA, 2012.

(c) 2018 by the authors. Licensee MDPI, Basel, Switzerland. This article is an open access article distributed under the terms and conditions of the Creative Commons Attribution (CC BY) license (http:/ / creativecommons.org/licenses/by/4.0/). 